\title{
Utilizing Morphological Correlators for Device Performance to Optimize Ternary Blend Organic Solar Cells Based on Block Copolymer Additives
}

\author{
Dylan Kipp \\ Department of Chemical Engineering, \\ University of Texas at Austin, Austin, TX 78712 \\ Olga Wodo \\ Materials Design and Innovation Department, \\ Mechanical and Aerospace Engineering Department, \\ University of Buffalo, Buffalo, NY 14260 \\ Baskar Ganapathysubramanian \\ Department of Mechanical Engineering, \\ Iowa State University, Ames, IA 50011 and \\ Department of Electrical and Computer Engineering, \\ Iowa State University, Ames, IA 50011 \\ Venkat Ganesan \\ Department of Chemical Engineering and Institute \\ for Computational and Engineering Sciences, \\ University of Texas at Austin, Austin, TX 78712*
}

(C) 2016. This manuscript version is made available under the Elsevier user license http://www.elsevier.com/open-access/userlicense/1.0/ 


\begin{abstract}
In a recent work, we studied donor-acceptor blend based organic photovoltaics and, by utilizing a combination of morphology simulations and device modeling, demonstrated that block copolymer compatibilizers with appropriately selected energy levels can be used in such systems to give rise to highly efficient devices that, in some cases, can even outperform idealized morphologies. In the present study, we probe whether morphological simulations can be used in conjunction with simple morphological descriptors as a means to screen the performance characteristics of such energy cascade based ternary blend devices as predicted using device-level simulations. Towards this objective, we present results from different parameter combinations to demonstrate that the domain size, percolation ratio, tortuosity of domains, and concentration gradient at the interface between donors and acceptors correlate strongly with the device performance of such ternary blend systems. Subsequently, we present extensive parameter studies where we simultaneously vary the blend composition, the degree of polymerization of the donor homopolymer, and the acceptor composition of the donor- $b$-acceptor block copolymer to identify blend formulations that give rise to such optimal morphological and device characteristics. Finally, we demonstrate that, while the overall device performance depends on a combination of morphological factors, the morphological descriptors identified in our work may help identify promising blend formulations.
\end{abstract}




\section{INTRODUCTION}

Typical organic photovoltaic bulk heterojunction (BHJ) cells are fabricated either by mixing conjugated polymer donors with acceptor $\mathrm{C}_{60}$ molecules (or their derivatives) or by blending donor and acceptor conjugated polymers. ${ }^{1-4}$ Often, such components are either immiscible or partially miscible, which leads to phase separation into domains, kinetically-trapped metastable states, ${ }^{5}$ and a degradation of the device performance with thermal/solvent annealing. ${ }^{6-15}$ As a result, there has arisen significant interest in strategies that can enhance the long-term stability of the devices.

In the above context, recently, the use of block copolymer (BCP) compatibilizers in donor-acceptor blends has emerged as a popular strategy to improve the device stability of organic photovoltaics (OPV). Pioneering experiments by Sivula et al. ${ }^{16}$ demonstrated that a block copolymer composed of poly(3-hexylthiophene) (P3HT) repeat units in one segment and fullerene pendants in the other can be used as a compatibilizer for P3HT:PCBM based OPVs. They demonstrated that the resulting device exhibited no domain coarsening or degradation in device properties even after annealing over a period of ten hours. A number of subsequent studies have demonstrated similar enhancements in long-term stability and device performance through the use of BCP compatibilizers in a variety of donor-acceptor based BHJ OPVs.

In recent studies, ${ }^{17-20}$ we demonstrated that BCP additives can serve a role that extends beyond compatibilization; namely, BCPs can be utilized in BHJ OPVs to actively modulate both the mesoscale morphology and the interfacial properties. We demonstrated that the addition of BCP compatibilizers to donor/acceptor blends can be used to create equilibrium bicontinuous morphologies with nanoscale domain sizes and percolating donor and acceptor

pathways. ${ }^{17-19}$ In a subsequent work, we demonstrated that, if the energy levels of the BCP are chosen appropriately for such ternary blend systems, an "energy cascade" can be formed at the interface between donor and acceptor domains. ${ }^{20}$ Such cascaded heterojunctions can be tuned to stabilize charges away from the interface thereby reducing charge recombination and improving the device efficiency (see Figure 1). The resulting device performance of such ternary blends reflect the combined influence of morphology and energy cascades, and, in 




FIG. 1. Energy cascades reduce charge recombination and increase device performance by creating a spacially-ordered distribution of site energies in the vicinity of the interface. Charges relax in this energy landscape thereby allowing the system energy to decrease despite the charges escaping their mutual coulombic attraction.

some instances, can be made to outperform even idealized columnar morphologies proposed for bulk heterojunction devices. ${ }^{20}$

Despite the successes reported in our studies, donor-acceptor-BCP based ternary blend mixtures are confronted with a vast parameter space that renders the design and optimization of such systems a complex and highly multidimensional problem. ${ }^{17,19}$ Indeed, the chemistry and photophysical properties of the chosen donors, acceptors, and BCPs, the relative molecular weights of the components, and the volume fraction of the different components constitute some of the many parameters that can be tuned by experimentalists. As a consequence, a trial-and-error approach incorporating the synthesis and characterization of many different blend formulations with the objective to optimize the device peformance is expected to be an intractable task. Hence, there is a need for strategies that can help narrow the parameter space by screening different formulations and eliminate the nonviable/underperfoming (in device performance) blends in favor of systems that exhibit desirable characteristics.

In the above context, we note that many previous studies have suggested a strong correlation between the morphology of bulk heterojunction solar cells and the resulting device performance. $^{21-27}$ Indeed, the life-time of the photogenerated excitons is believed to be of the order of pico/nanoseconds. Hence, morphologies presenting maximum donor-acceptor interfacial area and domain sizes on the order of $10 \mathrm{~nm}$ are expected to facilitate efficient charge carrier generation. Further, once the exciton dissociates into electrons and holes, the charge carriers require continuous morphological pathways to the respective electrodes. Not 
surprisingly, less tortuous morphologies that exhibit better connectivity to electrodes display better device performance. Motivated by such experimental findings, in an earlier work, ${ }^{28,29}$ we identified a suite of morphology measures/descriptors that exhibited a direct correlation to the device characteristics resulting in experiments ${ }^{30}$ and computer simulations. ${ }^{29,31}$ We suggested that such measures can be utilized to evaluate and compare different donor/acceptor morphologies without the need to conduct time consuming current-voltage device measurements or simulations. Moreover, such measures can also be employed in conjunction with computer simulations capable of predicting active layer morphologies and thereby provide an in-silico approach to screen the viability of devices constructed from different blend formulations.

While the morphological descriptors discovered in our earlier work ${ }^{28-30}$ relate to general donor-acceptor based BHJ devices, such measures do not make allowance for the changes in the photophysical properties of the device active layer that may result from the incorporation of a third blend component. For instance, in the context of our recent study on ternary blends, ${ }^{20}$ we found that the morphological characteristics alone may not suffice to capture the device characteristics in the presence of energy cascades induced by a third component. Instead, as a consequence of the photoelectronic characteristics of the BCP compatibilizers, the peak device performance was shown to be determined by the combined influence of both the morphological and interfacial properties of the resulting blends. Motivated by such findings, in this work we seek to address whether the earlier-identified suite of morphologydevice correlations can be expanded to account for the energy levels of the third component and the interfacial energy cascades that may result in such OPV devices. The discovery of such measures will provide a means to screen different ternary blend formulations based on their morphologies and thereby identify the blends that hold the most promise for device characteristics.

In this article, we present the results of a two part work motivated by the above considerations. In the first part, we utilize Single Chain in Mean Field (SCMF) simulations to generate morphologies based on the homopolymer donor + PCBM acceptor + donor- $b$ acceptor model system. We utilize graph-based methods to calculate a host of descriptors that characterize the mesoscale morphology and the interfacial properties of our example 
morphologies. We then probe the correlation of these descriptors to device performance characteristics as predicted by kinetic Monte Carlo (KMC) device simulations. We find that the set of morphological correlators for device performance can ultimately be reduced to a small set of descriptors that include new measures to account for the interfacial energy cascade effects. In the second part, we conduct studies over a much larger range of parameters to identify ternary blend formulations that optimize these descriptors and thereby furnish design rules for ternary blend organic solar cells based on the BCP compatibilizers. We use such findings to suggest a means by which screening of different formulations for device performance can be effected.

While much of the work detailed in the following sections relate to ternary blends based on BCPs and the specific parameters we adopt for such systems, we propose that the qualitative aspects of our findings are quite general and apply equally well to other classes of ternary blend systems. For instance, we note that there has arisen significant interest in organic ternary blends containing either an additional electron donor or acceptor material to enhance the absorption wavelengths compared to the primary donor-acceptor mixture. ${ }^{32-38}$ With appropriate adaptation, the findings of our work and the strategy proposed can also be used to address the coupling between morphology and interfacial properties in such contexts.

The rest of the article is arranged as follows: In Section II, we briefly discuss the methodological aspects underlying the simulations of morphology and device properties. Since the framework we adopt in this regard is identical to the framework used in our prior work, to maintain brevity, we present only the most salient details here. In such a context (Section IIB), we discuss the morphological descriptors we deploy and propose new measures that account for the interfacial characteristics in ternary blends. In Section III, we present the results in four parts. In Section III A, we present results for the case of ternary blends with energy levels of the BCP chosen to be identical to those of the donor and acceptor. In this case, there is an absence of energy cascade effects, and our results demonstrate that the morphological descriptors discovered in our previous work serve to capture the device performance of such ternary blends. In Section III B, we study the case where the energy levels of the BCP differ from those of the donor and acceptor thus leading to an energy cascade. In such a case, we demonstrate that additional interfacial measures are necessary to understand 
the device performance. In Section III C, we present results that demonstrate the influence of the physicochemical parameters of the ternary blend upon the different morphological and interfacial descriptors. Such an analysis serves to identify some design rules that can help optimize the general formulation of ternary blends based on BCPs. Finally, in Section III D, we discuss the optimization of device performance and the manner in which the results deduced in Sections III A - III C can enable such an objective.

\section{SIMULATION METHODS}

We use a combination of morphology and device-level simulation methods to investigate the influence of BCP compatibilizers on the morphology, interfacial properties, and device performance of organic solar cells. Specifically, we utilize SCMF and KMC simulations to respectively predict the equilbrium morphology and current-voltage behavior of our ternary blend system. These two methods are coupled in that the morphologies and interfacial characteristics predicted by the SCMF simulations are input into the KMC simulations. We then employ a graph-based method to generate a suite of morphology descriptors that characterize the SCMF morphologies. Finally, we correlate these descriptors with the device performance characteristics as predicted using KMC simulations that explicitly account for energy cascades. The details of our SCMF, KMC, and graph-based calculation methods are

all detailed in previous works, ${ }^{28,39,40}$ and, hence, we present only a brief description of the model details and the input parameter values used therein.

\section{A. Model System}

The model system we consider is a ternary blend of homopolymer donors (D1), PCBM acceptors (A1), and donor-b-acceptor block copolymers (D2-b-A2). For the morphology simulations, we utilize a Flory-Huggins interaction parameter, $\chi_{i j}$, to quantify the strength of compositional interactions between each of the individual blend components (A1, A2, D1, and D2). We consider the case of an ideal compatibilizer additive, where the D2 and A2 blocks of the BCP are designed to mix with the D1 and A1 components, respectively. 
Specifically, we set $\chi=\chi_{A 1 D 1}=\chi_{A 1 D 2}=\chi_{A 2 D 1}=\chi_{A 2 D 2}$ and $\chi_{A 1 A 2}=\chi_{D 1 D 2}=0$. In our morphology simulations, the BCP distributes preferentially to the interface between donor and acceptor domains (a finite concentration of BCP also distributes to the bulk of the donor and acceptor domains) where it reduces the interfacial tension (thereby yielding nanoscale domain sizes) and gives rise to a concentration gradient of A1, A2, D1, and D2 components (thereby yielding energy cascades). Although our selected set of $\chi_{i j}$ 's most closely resembles the case of the "ideal" BCP compatibilizer, we have previously demonstrated that the predictions utilizing this parameter set are qualitatively similar to those seen for different donor and acceptor molecular chemistries, given that the interactions between the components place the BCP at the interface in a similar fashion. ${ }^{19}$

Beyond the specification of the chemistries of the donor, acceptor, and BCP (which fix the $\chi_{i j}$ 's), our system is characterized by four other free parameters: i) the volume fraction of $\mathrm{BCP}$ in the blend $\left(\phi_{B C P}\right)$, (ii) the composition of the BCP $(f$, which denotes the acceptor fraction), (iii) a parameter that quantifies the degree of polymerization of the homopolymer relative to the degree of polymerization of the $\mathrm{BCP}$ (denoted $\beta$ ), and iv) the parameter $\triangle H O M O=H O M O_{D 2}-H O M O_{D 1}$, which quantifies the energy offset between the highest occupied molecular orbital of the homopolymer $\left(H O M O_{D 1}\right)$ and the highest occupied molecular orbital of the donor block of the $\mathrm{BCP}\left(\mathrm{HOMO}_{\mathrm{D} 2}\right)$. As most high efficiency devices are based on equal or nearly-equal amounts of donors and acceptors, we perform simulations based on equal volume mixtures of donors and acceptors, but modulate the parameters $\phi_{B C P}$ and $f$. Accordingly, blend formulations with higher $\phi_{B C P}$ and $f$ have higher ratios of $\phi_{A 2} / \phi_{A 1}$ in the blend (where $\phi_{i}$ indicates the blend composition of component $i$ ), although the sum $\phi_{A 1}+\phi_{A 2}$ is a constant. The parameter $\beta=\left(N_{\text {homo }}-N_{B C P}\right) / \min \left(N_{\text {homo }}, N_{B C P}\right)$ quantifies the molecular weight of the homopolymer relative to the molecular weight of the BCP where $N_{B C P}$ and $N_{\text {homo }}$ indicate the degrees of polymerization of the BCP and homopolymer, respectively. In this terminology, the homopolymer has a larger (smaller) degree of polymerization compared to the $\mathrm{BCP}$ for $\beta>0(\beta<0)$. We set $N_{B C P}=20$ and change $N_{\text {homo }}$ to effect changes in $\beta$. Finally, we set $\chi N_{B C P}=30$ for all cases.

Organic photovoltaics are typically based on fully-conjugated polymer donors. ${ }^{41}$ The conjugated backbone of such donors often endows the polymer with an inherent degree of semi- 
flexibility. Moreover, these donors often undergo different kinds of chain-packing interactions (for instance, $\pi-\pi$ stacking or liquid crystalline ordering). Experiments have demonstrated that both the semiflexibility and the chain-packing of such donor materials strongly influence the phase behavior of single component donor- $b$-acceptor BCPs. ${ }^{42-46}$ In a previous work, we developed a model of such BCPs and generated equilibrium phase diagrams that displayed good agreement with the experiments. ${ }^{47}$ However, when we expanded this model to treat the case of the ternary blends of interest in this work, we found that the influence of the donor semiflexibility and chain-packing effects on the equilibrium phase behavior was weak except at very high BCP loadings (where the phase behavior began to closely resemble that of the single-component BCP system) ${ }^{39}$ Considering these previous results, in this work, we choose to study a model of the donors that treats the chains as fully flexible and we implement only isotropic interactions (no chain packing effects).

We set $H O M O_{D 1}=-5.2 \mathrm{eV}$ and $L U M O_{A 1}=-4.2 \mathrm{eV}$ (where $L U M O_{i}$ denotes the lowest unoccupied molecular orbital of component $i$ ) in order to match the experimentally measured energy levels of commonly studied polymer donors and PCBM acceptors such as P3HT, PTB7, and PC61BM. ${ }^{48,49}$ We vary the energy levels of the BCP to create an energy cascade at the interface between donors and acceptors (see Figure 2). In a previous study, we demonstrated that donor energy cascades (with $H O M O_{D 2} \neq H O M O_{D 1}$ ) generally have a stronger influence on the device properties than acceptor energy cascades (with $L U M O_{A 2} \neq$ $\left.L U M O_{A 1}\right)$ due to a sharper concentration gradient of donors at the interface. ${ }^{20}$ Accordingly, in this work, we focus only on donor cascades and set $L U M O_{A}=L U M O_{A 1}=L U M O_{A 2}$ in all cases. However, we do consider different values of $\mathrm{HOMO}_{\mathrm{D} 2}$ and, therefore, different $\triangle H O M O$. In the case of $\triangle H O M O=0 \mathrm{eV}$, the D2 and D1 donors are indistinguishable from one another, and the only influence of changing $\phi_{B C P}$ and $f$ is on the equilibrium morphology. In contrast, in the case of $\triangle H O M O \neq 0 \mathrm{eV}$ and $\phi_{B C P} \neq\langle 0,1\rangle$, an energy cascade forms due to the concentration gradient of donors across the cross section of donor domains.

While the SCMF simulations capture the equilibrium concentration profile across domains, the KMC simulations serve to account for the influence of the resulting energy cascades upon the device properties. ${ }^{20}$ Specifically, in the KMC simulations, we select the oc- 


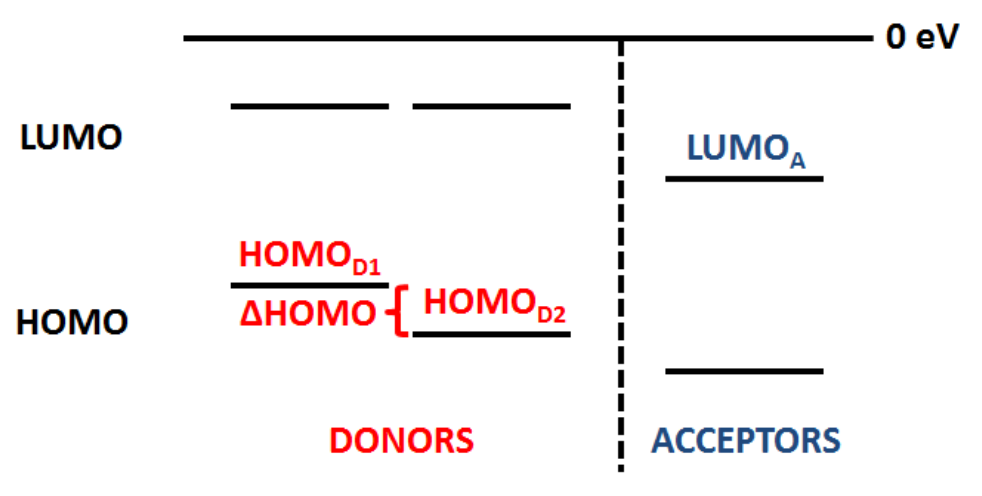

FIG. 2. Schematic of the HOMO and LUMO energy levels for the model system of interest. The HOMO of the donor block of the BCP is shifted relative to the HOMO of the homopolymer according to $\triangle H O M O=H O M O_{D 2}-H O M O_{D 1}$, giving rise to an energy cascade on the donor side of the donor/acceptor interface. In contrast, the acceptor block of the BCP and the PCBM are set to have the same energy levels $\left(L U M O_{A}=L U M O_{A 1}=L U M O_{A 2}\right)$, and there is no energy cascade on the acceptor side of the interface.

cupation energy of each donor site from a Gaussian density of states with an expected value equal to the composition-averaged $\mathrm{HOMO}$ of $\mathrm{D} 1$ and $\mathrm{D} 2$ at that donor site $(H O M O=$ $\left.\left(\phi_{D 1} H O M O_{D 1}+\phi_{D 2} H O M O_{D 2}\right) /\left(\phi_{D 1}+\phi_{D 2}\right)\right)$. In addition, $\triangle H O M O \neq 0 \mathrm{eV}$ leads to a tunable field across the device active layer calculated as $F=F_{\text {applied }}+H O M O_{b l e n d}-L U M O_{b l e n d}$ where $F_{\text {applied }}$ is the applied electric field and $H O M O_{\text {blend }}\left(L U M O_{\text {blend }}\right)$ is the blend-averaged HOMO of donors (blend-averaged LUMO of acceptors). These methods for calculating the distribution of donor site energies and F match the "organic alloy model" for well-mixed two donor systems recently proposed by Klyabich et al. ${ }^{50}$

\section{B. Morphological Descriptors}

In previous works, ${ }^{29-31}$ Wodo et al. developed and used graph-based approaches to extract morphology descriptors from morphologies (both experimental morphologies as well as numerically generated morphologies). These morphology descriptors were then correlated with device performance of bulk heterojunction organic solar cells. The underlying basis for the framework to efficiently extract morphology descriptors (like tortuosity, interfacial area, domain sizes) is the equivalence between a morphology (represented in a discrete or 
pixel form, see Figure 5) and a weighted, colored, undirected graph. ${ }^{29-31}$ In particular, each pixel (or voxel) becomes a node in the graph with a color label denoting its phase (white represents acceptor material and black represents donor material). Each node is connected to its neighboring nodes through edges. Each edge is labeled with a weight that corresponds to the physical distance between the nodes (see Figure 3). Once the morphology is represented as an equivalent graph, all morphology traits can be expressed in terms of properties of the graph, with very efficient algorithms from graph theory available to rapidly evaluate these descriptors. Examples include using the shortest graph distance algorithm to identify charge transport pathways for electrons (through the acceptors) and holes (through the donor), as well as using the graph connected components algorithm to identify simply connected domains of donors and acceptors, and quantify which domains are connected to the electrodes. For each of the physical subprocesses underlying the photovoltaic process several simple morphological descriptors can be defined to serve as a performance indicator. For instance, the efficiency of exciton dissociation was linked with the distance from any donor site to the interface, a result that can be understood by noting that excitons that form in the center of large domains are likely to decay before they find an interface. The efficiency of charge separation was linked to both the connectivity of domains and tortuosity of paths within the morphology, since charges that form in islands unconnected from their partnered electrode are unable to be collected, and, moreover, tortuous pathways create bottlenecks where charges are more likely to buildup and recombine. We define tortuosity as a ratio of the length of the shortest path through the complex morphology (assuming that paths can consist of only one type of phase) to the length of the straight and direct path to the electrode (as there are no obstacles). For such a definition, the perfect path is characterized by tortuosity one, as the actual path though the complex morphology is the same as the straight rising path. With such definition of tortuosity, we can filter straight rising paths $(\tau=1)$ from more tortuous the path $(\tau>1)$. The evaluation of the tortuosity measure has been previously utilized to analyze charge transport characteristics in both experimental and synthetic morphologies. ${ }^{31,51}$

Motivated by the above findings, in this work, we utilize the graph-based approach to calculate morphological descriptors to characterize the ternary blend morphologies that result 

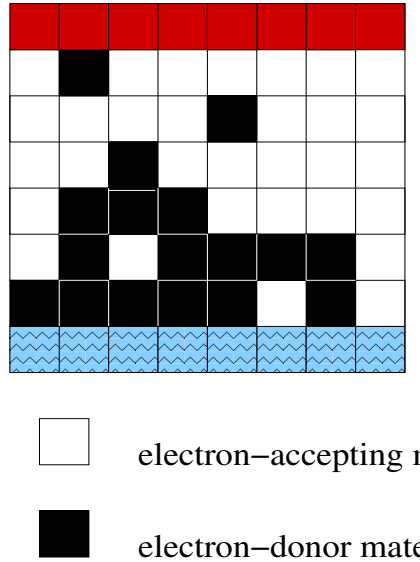

electron-accepting material

electron-donor material

anode

cathode

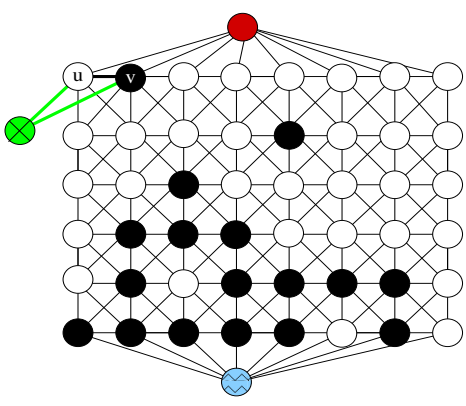

anode

Q cathode

$\otimes$ interface

FIG. 3. A schematic of the implementation of the graph-based analysis of the morphologies resulting in our simulations.

from the SCMF simulations. ${ }^{29,30}$ We calculate the following domain descriptors to describe the size, tortuosity, and connectivity of donor and acceptor domains:

1. $D(\mathbf{n m}):$ The distance from a donor node to the nearest interface between donors and acceptors. This is a local quantity that differs from node to node.

2. $\langle D\rangle(\mathbf{n m})$ : The quantity $D$ averaged over all donors node. This is a single quantity that describes the average size of donor domains.

3. $X$ : The connectivity or percolation ratio of donor domains to the anode $\left(X_{d o n}\right)$ or acceptor domains to the cathode $\left(X_{a c c}\right)$. This is simply the ratio of the number of connected donor or acceptor node to the total number of donor or acceptor node.

4. $\tau$ : The tortuosity of the charge transport pathway from a donor node to the anode $\left(\tau_{d o n}\right)$ or from an acceptor node to the cathode $\left(\tau_{a c c}\right)$. Similar to $D$, this is also a local quantity, and is calculated as the ratio of two distances: i) the shortest distance (computed using the graph shortest path) from a donor (or acceptor) node to its respective electrode via only donor (or acceptor) node; ii) the shortest straight-line distance from the node to the electrode regardless of the surrounding distribution of 
donor and acceptor node. In this context, $\tau=1$ corresponds to a charge transport path for a node that is a straight and continuous pathway to the electrode, whereas $\tau>>1$ corresponds to charge transport path of a site with a highly tortuous pathway to the electrode. $\tau$ is not calculated for unconnected sites.

5. $\langle\tau\rangle$ : The quantity $\langle\tau\rangle$ averaged over all donor or acceptor sites $\left(\left\langle\tau_{\text {don }}\right\rangle\right.$ or $\left.\left\langle\tau_{a c c}\right\rangle\right)$ that are connected to their respective electrode.

The domain descriptors discussed above capture the influence of domain structure on device performance, and, hence, they are expected to be relevant both in the presence and in the absence of energy cascades arising from a third component.

To capture the influence of the energy cascades resulting from the presence of the third component (BCP), we hypothesize that additional morphological descriptors may be necessary. In a previous work, ${ }^{52}$ Groves studied the device performance of toy morphologies with step-like energy cascades at the interface between donors and acceptors. His morphologies consisted of donor domains, acceptor domains, and a cascade interlayer each with different energy levels. To characterize the structure of his energy cascades, he utilized two parameters: i) the HOMO edge offset between the donor and the cascade material, and ii) the width of the cascade interlayer between donors and acceptors. Groves found that the charge separation efficiency of the devices was strongly correlated to these two parameters, such that materials with larger HOMO offsets and thinner cascades led to higher charge separation efficiencies.

In comparison to Groves work, our work differs in two important ways: i) the structure of the energy cascade is not selected arbitrarily, but results from the output of morphological simulations, and, as a consequence, ii) the cascade is not step-like, but varies more gradually within the system. Since the energy levels of sites themselves are calculated based on the component compositions, we hypothesize that the efficacy of the energy cascade will correlate to the concentration profile in the vicinity of the interface. With this in mind, we calculate the following interfacial descriptors inspired by the work of Groves: ${ }^{52}$

1. $R=\phi_{D 1} /\left(\phi_{D 1}+\phi_{D 2}\right)$ : The ratio of D1 to sum D1 $+\mathrm{D} 2$ donors at a donor site. This is a local quantity. 
2. $R_{D}$ : The value $R$ averaged over all donor sites at a distance $D$ to the interface. Such a measure quantifies the variations of donor concentrations and, as a consequence of our model, the energy levels of the donors at different locations in the domains.

3. $\Delta R_{D}\left(\mathbf{n m}^{-1}\right)$ : The derivative $\partial R_{D} / \partial D$ at $D=0$. This quantity describes the slope of donor concentrations in the vicinity near the interface and is likely to serve as an indicator of the strength of the energy cascade.

4. $\Delta_{L}(\mathbf{n m})$ : The width of the interfacial region over which $R_{D}$ changes value. This quantity can be utilized as a measure of the width of the energy cascade. To calculate $\Delta_{L}$, we generate the $R_{D}$ profile based an SCMF simulation and identify $\Delta_{L}$ as the value of $D$ where $R_{D}$ reaches $95 \%$ of its bulk value $(D>>0)$.

\section{Single Chain in Mean Field Simulations}

SCMF is a simulation method that embeds the statistics of a self-consistent field theory (SCFT) for polymers ${ }^{53}$ into a Monte Carlo framework. ${ }^{54}$ Hence, the results of SCMF simulations are expected to be consistent with the SCFT except in-so-far as including the effects of thermal fluctuations. ${ }^{55,56}$ We utilize SCMF simulations to predict the equilibrium morphologies of our ternary blend system. Details of the methodology and its implementation for the system of the present study can be found in our earlier articles. ${ }^{17-20}$ In the present work, all the SCMF simulations were effected in three-dimensional space unless otherwise noted. In the case of two-dimensional SCMF simulations, the potential fields are averaged over only two directions, but the polymer chains are allowed to move in fully three-dimensional space.

\section{Kinetic Monte Carlo Simulations}

We utilize KMC simulations to predict the device performance of ternary blend morphologies that emerge from SCMF simulations. Specifically, we utilize the KMC framework from our previous article, ${ }^{40}$ except that we eliminate charge injection at the electrodes from the event list and set all energy barriers for charge extraction to large negative values. This 
treatment effectively eliminates charge buildup at the interface with the electrodes and allows us to more easily identify the influences of morphology and energy cascades on device performance. We utilize the same set of parameters from our previous work (see Table I), which is suitable for treating conjugated polymer donor/PCBM acceptor blends such as $\mathrm{P} 3 \mathrm{HT}+\mathrm{PCBM} \cdot{ }^{20,57}$

TABLE I. Kinetic Monte Carlo Parameters

\begin{tabular}{|c|c|c|}
\hline Parameter, symbol & Value & Unit \\
\hline \multicolumn{3}{|l|}{ Exciton } \\
\hline Exciton Photogeneration Rate of Homopolymer Donor, $w_{p h o t o, D 1}$ & 900 & $n m^{-3} s^{-1}$ \\
\hline Exciton Photogeneration Rate of PCBM Acceptor, $w_{\text {photo }, A 1}$ & 0 & $n m^{-2} s^{-1}$ \\
\hline Exciton Hopping Attempt Frequency x Localization Radius` $6, w_{e} R_{0}^{6}$ & $2 \times 10^{12}$ & $n m^{6} s^{-1}$ \\
\hline Exciton Recombination Rate, $w_{e r}$ & $2 \times 10^{9}$ & $s^{-1}$ \\
\hline \multicolumn{3}{|l|}{ Electron and Hole } \\
\hline Electron Mobility, $\mu_{e}$ & $3 \times 10^{11}$ & $n m^{2} V^{-1} s^{-1}$ \\
\hline Hole Mobility, $\mu_{h}$ & $5.8 \times 10^{10}$ & $n m^{2} V^{-1} s^{-1}$ \\
\hline Charge Recombination Rate, $w_{c r}$ & $1 \times 10^{9}$ & $n m^{2} V^{-1} s^{-1}$ \\
\hline \multicolumn{3}{|l|}{ Polymer Medium } \\
\hline $2 \times$ Polaronic Binding Energy, $E_{R}$ & 0.187 & $e V$ \\
\hline Standard Deviation of Gaussian Density of States, $\sigma$ & 0.062 & $e V$ \\
\hline Relative Permittivity, $\epsilon$ & 3.5 & \\
\hline Lattice Constant, $a_{0}$ & 3 & $n m$ \\
\hline HOMO of Homopolymer Donor, $H O M O_{D 1}$ & -5.2 & $e V$ \\
\hline LUMO of PCBM Acceptor, $L U M O_{A 1}$ & -4.2 & $e V$ \\
\hline
\end{tabular}

\section{RESULTS AND DISCUSSION}

\section{A. Identifying the Correlations Between Device Performance and Morphology in} the Absence of Energy Cascades

In our earlier work, ${ }^{29-31}$ we considered morphologies arising from binary donor/acceptor systems and used drift-diffusion models to characterize their device properties. ${ }^{58-61}$ Such a study identified the morphological domain descriptors discussed in Section II B. As a first step towards the objectives of the present work, we begin by considering the ternary blend morphologies arising in the absence of energy cascades and use KMC simulations to 
characterize their device characteristics. Using such results, we seek to verify whether the morphological measures deduced in our earlier work apply equally well to the morphologies and device characteristics resulting in the present study.

Figure 4 displays the device performance characteristics (Figure 4A) and domain descriptors (Figure 4B) for a set of example ternary blend morphologies obtained by varying the volume fraction of the block copolymer in the blend, $\phi_{\mathrm{BCP}}$. We observe that increasing $\phi_{B C P}$ leads to a monotonically increasing exciton dissociation efficiency, $\eta_{E D}$, and a monotonically decreasing fill factor, $F F$. In contrast, with increasing $\phi_{B C P}$, the charge separation efficiency, $\eta_{C S}$, is seen to first increase to a maximum and then decrease thereafter.

To rationalize the above results, we consider the behavior of the morphological domain descriptors with varying $\phi_{\mathrm{BCP}}$ (Figure $4 \mathrm{~B}$ ). We observe that increasing $\phi_{B C P}$ leads to a monotonically decreasing (average) domain spacing, $\langle D\rangle$. For these conditions, the donor domains were seen (results not shown) to percolate $\left(X_{\text {don }}=1\right)$ and exhibit minimal tortuosity $\left(\left\langle\tau_{d o n}\right\rangle \approx 1\right)$ for all $\phi_{B C P}$. In contrast, from Figures $4 \mathrm{~B}$ and Figure 5 , it can be seen that the acceptor domains exhibit a range of $X_{a c c}$ and $\left\langle\tau_{a c c}\right\rangle$. For $\phi_{B C P}=0.0$, the morphology is characterized by a collection of acceptor islands unconnected from the cathode $\left(X_{a c c}=0.34\right)$. Electrons are expected to become "trapped" in such acceptor islands thus leading to charge build-up and increased charge recombination, which is consistent with small $\eta_{C S}$ observed for such conditions. Increasing $\phi_{B C P}$ from $\phi_{B C P}=0$ to $\phi_{B C P}=0.1$ yields an increase in $X_{a c c}$ to $X_{a c c}=1.0$ and a corresponding increase in $\eta_{C S}$. However, $\eta_{C S}$ is seen to remain well below its maximum value of unity. To understand such a result, we observe that the morphology for $\phi_{B C P}=0.1$, for instance, is characterized by $\left\langle\tau_{a c c}\right\rangle=1.42$, which suggests the presence of tortuous acceptor domains. In such morphologies, electrons must travel extended distances, sometimes either perpendicular or counter-parallel to the field, in order to escape their partnered charge. Such considerations rationalize the values observed for $\eta_{C S}$. Consistent with such argument, increasing $\phi_{B C P}$ from $\phi_{B C P}=0.1$ to $\phi_{B C P}=0.2$ yields a reduction in $\left\langle\tau_{a c c}\right\rangle$ and a related increase in $\eta_{C S}$. From $\phi_{B C P}=0.2$, further increase of $\phi_{B C P}$ yields increasingly smaller $\langle D\rangle$ without a significant change of either $X_{a c c}$ or $\left\langle\tau_{a c c}\right\rangle$. The $\eta_{C S}$ is seen to decrease gradually matching the trends displayed by $\langle D\rangle$. In sum, $\eta_{C S}$ is seen to increase more sharply with increasing $X$ or decreasing $\langle\tau\rangle$, whereas it is seen to exhibit a 

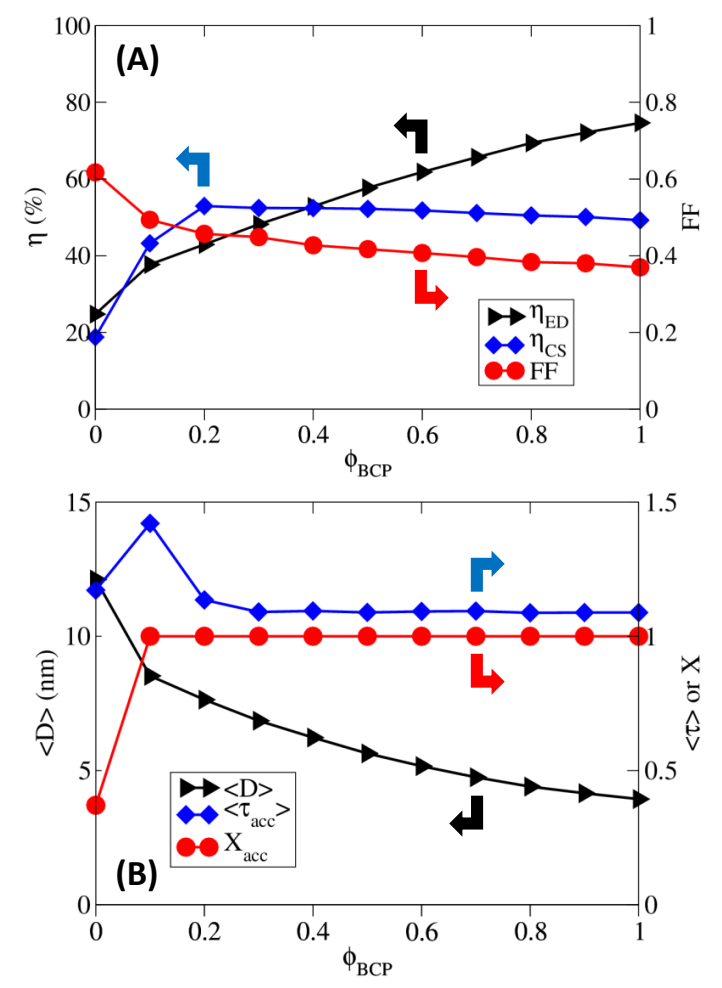

FIG. 4. Device performance and domain descriptors of ternary blends with $\beta=0.0, f=0.5$, and $\triangle H O M O=0 \mathrm{eV}$ (no energy cascades) for varying volume fractions of block copolymer in the ternary blend, $\phi_{\mathrm{BCP}}:(\mathrm{A})$ Exciton dissociation efficiency, $\eta_{E D}$, charge separation efficiency, $\eta_{C S}$, under short-circuit conditions, and the fill factor, $F F$. (B) Morphological descriptors $\langle D\rangle,\left\langle\tau_{a c c}\right\rangle$, and $X_{a c c}$ displayed as a function of $\phi_{B C P}$.

more gradual dependence on $\langle D\rangle$. Finally, in Figure 4, it can be seen that although $\eta_{C S}$ clearly depends on both $X$ and $\langle\tau\rangle$ in addition to $\langle D\rangle$, the fill factor $F F$ and the exciton dissociation efficiencies $\left\langle\eta_{E D}\right\rangle$ are insensitive to $X$ and $\langle\tau\rangle$ (Figure $4 \mathrm{~A}$ ). Rather, $F F$ is seen to correlate directly with $\langle D\rangle$ while $\eta_{E D}$ is seen to inversely correlate with $\langle D\rangle$.

In our earlier work based on binary donor-acceptor mixtures and drift-diffusion models, ${ }^{29,30,58-61}$ we demonstrated that the efficiency of exciton dissociation was correlated to the size of donor domains, whereas the efficiency of charge separation was correlated to both the connnectivity and tortuosity of domains (in addition to the domain sizes). The results discussed in the present section are seen to be consistent with the earlier results and suggests that the morphological descriptors deduced in our prior work apply equally well for the understanding of the device characteristics of the ternary blend system in the absence of energy cascades. 



FIG. 5. Representations of ternary blend morphologies with $\beta=0, f=0.5$, and varying $\phi_{B C P}$ : (A) $\phi_{B C P}=0.0,(\mathrm{~B}) \phi_{B C P}=0.1,(\mathrm{C}) \phi_{B C P}=0.2,(\mathrm{D}) \phi_{B C P}=0.3$. Shown are the surfaces of the acceptor domains (the donor domains make up the empty space). The electrodes are located at the bottom and top of each morphology. The colors indicate the z-position in the film - they are utilized to guide the eye and to make the domain surfaces easier to differentiate.

\section{B. Identifying the Correlations Between Device Performance and Morphology in} the Presence of Energy Cascades

We now vary $\triangle H O M O$ to generate energy cascades of varying strength at the interface between donor and acceptor domains and investigate the associated influence on charge recombination. Here, our objective is to identify the manner in which the device performance in such cases correlate with the interfacial descriptors discussed in Section II B. We note that energy cascades form upon the condition that $\triangle H O M O \neq 0$ and $\phi_{B C P} \neq\{0,1\}$. In our previous work, ${ }^{20}$ we demonstrated that only energy cascades with $\triangle H O M O<0$ stabilize holes away from the interface and reduce charge recombination whereas energy cascades with $\triangle H O M O>0$ promote holes to the interface and increase charge recombination. ${ }^{20}$ Motivated by practical device considerations, we choose to study only the case of $\triangle H O M O<0$ in this work.

Figure 6 displays the device characteristics and Figure 7 depicts the corresponding in- 

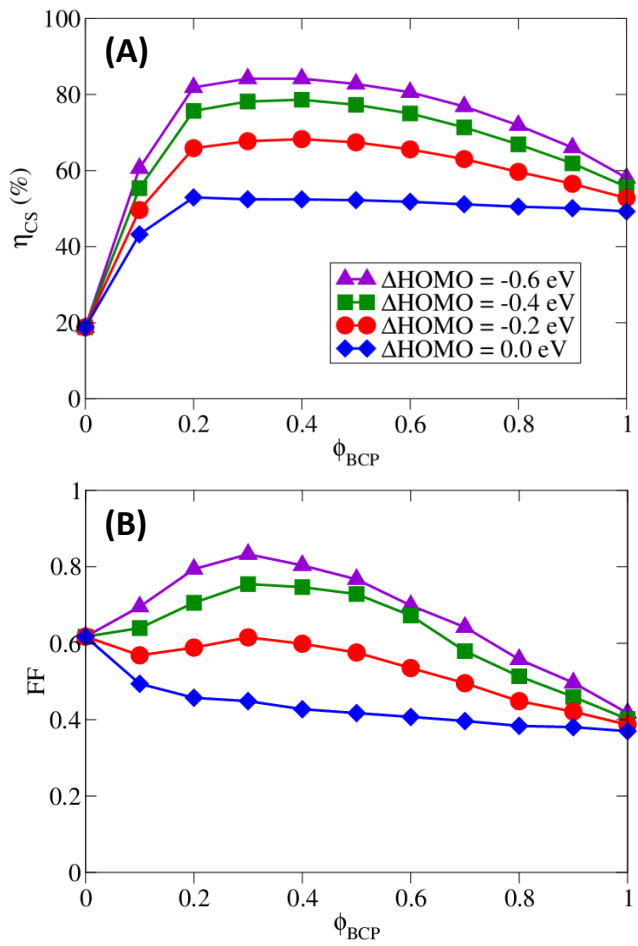

FIG. 6. Charge separation efficiency, $\eta_{C S}$, under short-circuit conditions, and the fill factor, $F F$, as a function of $\phi_{B C P}$ for ternary blends in the presence of energy cascades with different $\triangle H O M O$. The system parameters are $\beta=0.0$ and $f=0.5$.

terfacial descriptors for a ternary blend system identical to that discussed in the previous section, however, with a nonzero $\triangle H O M O$ arising from the differences in the energy levels of the $\mathrm{BCP}$ and the donor. In Figure 6, we observe that decreasing $\triangle H O M O$ to more negative values yields stronger cascades and increasingly larger $\eta_{C S}$ and $F F$. However, the relative increases in $\eta_{C S}$ and $F F$ with increasing magnitudes of $\triangle H O M O$ are seen to diminish as we approach the maximum limits corresponding to $\eta_{C S}=1.0$ and $F F=1.0 .^{52}$ Interestingly, both $\eta_{C S}$ and $F F$ are seen to exhibit a peak value at an intermediate value of $\phi_{B C P}$. Moreover, for sufficiently negative values of $\triangle H O M O$, the volume fraction $\phi_{B C P}$ corresponding to the peak of $\eta_{C S}$ also corresponds to the peak $F F$ and is also seen to be relatively insensitive to the value of $\triangle H O M O$. Explicitly, $\eta_{C S}$ and $F F$ peak near $\phi_{B C P}=0.3$ for each of $\triangle H O M O=-0.2 \mathrm{eV},-0.4 \mathrm{eV}$, and $-0.6 \mathrm{eV}$, but $\eta_{C S}$ peaks at $\phi_{B C P} \simeq 0.2$ whereas $F F$ peaks at $\phi_{B C P}=0$ for $\triangle H O M O=0.0 \mathrm{eV}$. 
In an earlier work, Groves considered the model case of step-like energy cascades and identified that the efficacy of energy cascades for reducing charge recombination decreases with increasing width of the cascade. ${ }^{52}$ In our system, we recall that as a consequence of the presence of donors and block copolymers throughout the morphology, there is a distribution of donor site energies that characterize the energy cascade. In section II B we proposed the measures $R_{D}$ and $\Delta_{L}$ as a means to characterize such variations. Hence, as a first step to understand the trends observed in Figure 6, we turn to a consideration of $R_{D}$ and its dependence on $D$ for different $\phi_{B C P}$.

Figure 7A displays $R_{D}$ vs $D$ for varying $\phi_{B C P}$ while Figure $7 \mathrm{~B}$ depicts the corresponding measures $\Delta R_{D}$ and $\Delta_{L}$. In Figure $7 \mathrm{~A}$, we observe that $R_{D}$ exhibits a gradual increase from the interface $(D=0)$ to the bulk $(D>>0)$ for all $\phi_{B C P} \neq\langle 0,1\rangle$ (the decrease in $R_{D}$ seen for large $D$ in some cases is a result of the spurious influence of the second interface enclosing the domain). These results demonstrate that the donor concentration and the resulting energy cascades produced in ternary blend systems are not step-like, but rather, vary more gradually. The corresponding measures $\Delta R_{D}$ and $\Delta_{L}$ are seen to peak at intermediate, but differing $\phi_{B C P}$. Explicitly, $\Delta R_{D}$ is seen to exhibit a maximum near $\phi_{B C P}=0.3$, whereas $\Delta_{L}$ peaks near $\phi_{B C P}=0.6$.

By comparing the results of Figures $6 \mathrm{~A}$ and $6 \mathrm{~B}$ to Figure 7 , it is evident that $\eta_{C S}$ and $F F$ correlate very closely with $\Delta R_{D}$. Indeed, the maxima in both $\eta_{C S}$ and $F F$ were seen to occur (for nonzero $\triangle H O M O$ ) near $\phi_{B C P} \simeq 0.3$, which matches with the trends seen for $\Delta R_{D}$. Overall, these results can be understood at a physical level by noting that $\Delta R_{D}$ serves to capture more quantitatively the strength of the energy cascade, which is expected to be the main factor determining the charge separation efficiencies and fill factor. In contrast, $\Delta_{L}$ is a measure which is more sensitively dependent on the length scale of variations of $R_{D}$, but does not embody information on the magnitude of such variations.

Together, the results of Figures 4-7 reveal the critical morphological and interfacial aspects that determine the device performance for the ternary blend system. Explicitly, $\eta_{E D}$ correlates only with the average domain spacing $\langle D\rangle$ and does not depend on the presence of the energy cascades resulting from a third component. In contrast, $\eta_{C S}$ correlates with each of $X,\langle\tau\rangle,\langle D\rangle$, and, in the presence of energy cascades, the measure $\Delta R_{D}$. The fill 

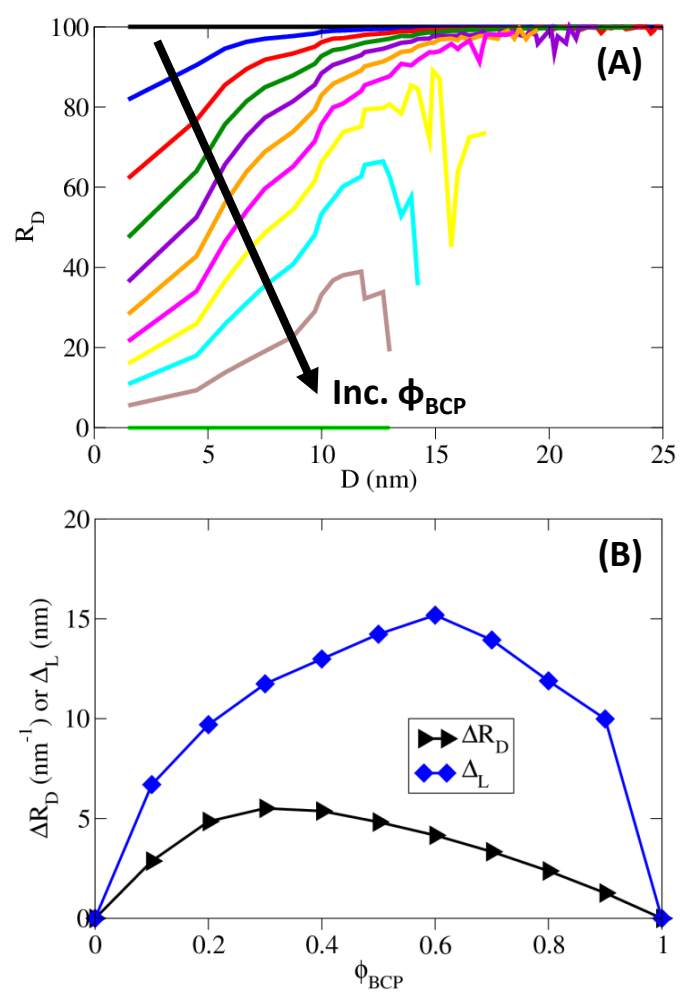

FIG. 7. Interfacial descriptors for BCPs with $\beta=0$ and $f=0.5$ : (A) Results corresponding to different $\phi_{B C P}$ on the range of $\phi_{B C P}=\{0.0,1.0\}$ in steps of $\Delta \phi_{B C P}=0.1$, and the arrow indicates the direction of increasing $\phi_{B C P}$; (B) $\Delta R_{D}$ and $\Delta_{L}$ displayed as a function of $\phi_{B C P}$.

factor, $F F$, correlates with $\langle D\rangle$ and (for $\triangle H O M O \neq 0$ ) $\Delta R_{D}$. In more detailed terms, it was evident that $X_{a c c}=X_{d o n}=1$ and $\left\langle\tau_{a c c}\right\rangle \approx\left\langle\tau_{d o n}\right\rangle \approx 1$ is a requisite condition to maximize $\eta_{C S}$ (see Figure 4). Upon meeting this condition, $\eta_{C S}$ decreases only gradually with increasing $\langle D\rangle$ and remains near its peak value over a large range of $\langle D\rangle$. Hence, the target optimal morphology should incorporate, at minimum, all percolating donor and acceptor domains with minimal tortuosities in order to minimize charge recombination. Given that this condition is satisfied, the combination of $\eta_{E D}, \eta_{C S}$, and $F F$ is then expected to correlate only with the morphological descriptors $\langle D\rangle$ and $\Delta R_{D}$.

Based on the above conclusion, as a final step to this analysis, we now probe the correlations between device performance and morphology with the intent of identifying which of the measures $\langle D\rangle$ or $\Delta R_{D}$ has a stronger influence on the device performance characteristics. Figure 8 displays $\eta_{E D}, \eta_{C S}$, and $F F$ parametrically as a function of $\langle D\rangle$ and $\Delta R_{D}$ for select 

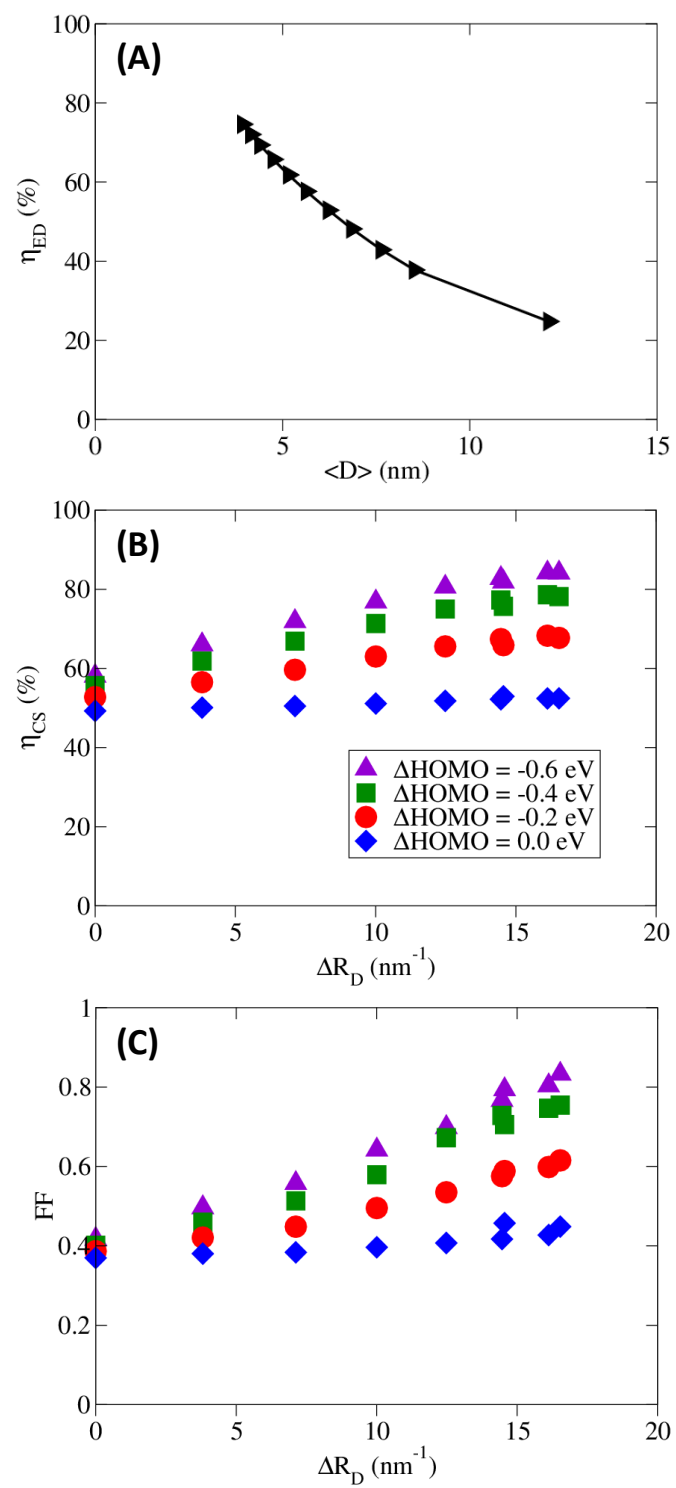

FIG. 8. Morphological correlators for device performance for select cases where $X_{a c c}=X_{d o n}=1$ and $\left\langle\tau_{a c c}\right\rangle \approx\left\langle\tau_{d o n}\right\rangle \approx 1$. (A) Exciton dissociation efficiency, $\eta_{E D}$ displayed as a function of $\langle D\rangle$; (b) The charge separation efficiency $\eta_{C S}$ under short-circuit conditions displayed as a function of $\Delta R_{D}$; (C) The fill factor, FF, displayed as a function of $\Delta R_{D}$. The BCP parameters are: $\beta=0$ and $f=0.5$.

cases where $X_{a c c}=X_{d o n}=1$ and $\left\langle\tau_{a c c}\right\rangle \approx\left\langle\tau_{d o n}\right\rangle \approx 1$. Consistent with our discussion in the context of Figure 4, in Figure 8A we observe an inverse correlation between $\eta_{E D}$ and $\langle D\rangle$. Since interfacial descriptors are not expected to influence $\eta_{E D}$, we do not consider variations of $\eta_{E D}$ in terms of $\Delta R_{D}$. 
Figures $8 \mathrm{~B}$ and $8 \mathrm{C}$ depict $\eta_{C S}$ and $F F$ for cases that vary simultaneously in $\Delta R_{D}$ and $\langle D\rangle$. The results for $\triangle H O M O=0.0$ pertain to the case wherein energy cascades are absent and the changes in $\eta_{C S}$ and $F F$ arise only from changes in $\langle D\rangle$. For such a case, it is seen that, while $\eta_{C S}$ and FF does vary, the magnitude of such changes are much smaller compared to the variations seen for non zero $\triangle H O M O$. Such results are consistent with those discussed in the context of Figure 4, wherein we saw that an increase in $\langle D\rangle$ resulted in only small changes in $\eta_{C S}$ and $F F$ (for the regime $X_{a c c}=X_{d o n}=1$ and $\left\langle\tau_{a c c}\right\rangle \approx\left\langle\tau_{d o n}\right\rangle \approx 1$ ). However, when $\triangle H O M O$ is nonzero, we observe that both $\eta_{C S}$ and $F F$ exhibit an almost linear increase with $\Delta R_{D}$. Since the $\langle D\rangle$ values are unchanged relative to the case of $\triangle H O M O=0.0$, we conclude that, for cases of non zero energy cascades, the influence of $\Delta R_{D}$ proves to be a much more significant factor relative to $\langle D\rangle$. These trends suggest that, for the purpose of optimizing $\eta_{C S}$ and $F F$ in situations with energy cascades, it may suffice to ignore the weak influence of $\langle D\rangle$ and consider $\Delta R_{D}$ as the main parameter to be targeted.

In sum, based on the results presented in Sections III A and III B, we identify $\Delta R_{D}$ as the main morphological quantity for optimizing $\eta_{C S}$ and $F F$ in ternary blend systems with energy cascades. In contrast, the average domain spacing $\langle D\rangle$ serves as the main target for optimizing $\eta_{C S}$ and $F F$ when energy cascades are absent and as the target for $\eta_{E D}$ in all cases. In the next section, we present results that use such findings as the basis to explore a wide range of parameters $\left(\beta, f\right.$, and $\left.\phi_{B C P}\right)$ in ternary blends based on BCP compatibilizers to identify those areas of the parameter space that yield the largest $\Delta R_{D}$ and the smallest $\langle D\rangle$. While the specific details of our results are dependent on the parameters used in our work, the simple design rules derived can enable the optimization of general ternary blend OPVs based on BCPs. Moreover, the results and discussion presented in the next section illustrate the general strategy by which the morphological descriptors can be integrated with morphology prediction tools. 


\section{Optimizing Morphological Descriptors Through Physicochemical Parameters}

of the Blend

For the studies presented in this section, we simulated equal volume mixtures of donor and acceptor in order to target $X_{a c c}=X_{d o n}=1$ and $\left\langle\tau_{a c c}\right\rangle \approx\left\langle\tau_{d o n}\right\rangle \approx 1$. Also, we utilized only $2 \mathrm{D}$ variation of the composition fields in the SCMF simulations in order to reduce the computational overhead. Since the $X$ and $\langle\tau\rangle$ from 2D simulations may not necessarily correspond to those characterizing 3D morphologies, we identified each 2D morphology explicitly as either a donor droplet, acceptor droplet, or lamella phase; 2D lamella phases are the most likely to yield $X=1$ and $\langle\tau\rangle \approx 1$ for 3D simulations, whereas 2D droplet morphologies are more likely to yield $X<1$ and/or $\langle\tau\rangle>1$. $^{17,19}$

Figure 9 displays $\Delta R_{D}$ for varying $\beta, f$, and $\phi_{B C P}$. Also shown are black lines that categorize the results based on the morphologies. We observe that acceptor droplets form for small $f$ and $\phi_{B C P}$, and that increasing either $f$ or $\phi_{B C P}$ leads to a transition from acceptor droplets to lamella morphologies and, then, from lamella to donor droplet morphologies. These transitions can be understood primarily as a consequence of changing the blend composition of acceptor A1. Indeed while the blend composition consists of a 1:1 ratio of donors and acceptors, for small $f$ and $\phi_{B C P}$, the acceptors in the blend are comprosed almost entirely of A1 since the blend composition of A2 is negligible. The A1 distributes preferentially to the acceptor domains, but, due to the entropy of such small molecules, also partially infiltrates into the donor domains. Accordingly, for small $f$ and $\phi_{B C P}$, the donor domains make up the continuous, larger portion of the active layer, and the minority acceptor domains form droplets in the continuous donor domain. Increasing either $f$ or $\phi_{B C P}$ reduces the blend composition of $\mathrm{A} 1$, thus increasing the cumulative volume of acceptor domains, leading first to a transition to lamella morphologies and then to a transition to donor droplets in a continuous acceptor domain. We note that, for those blend formulations that form acceptor droplet (donor droplet) morphologies, lamella morphologies could also be achieved by increasing (decreasing) the total blend composition of acceptors.

In the context of morphological descriptors, we observe that $\Delta R_{D}$ increases with increasing $\beta$. This trend can be rationalized by invoking the degree of swelling of the D2 block of 

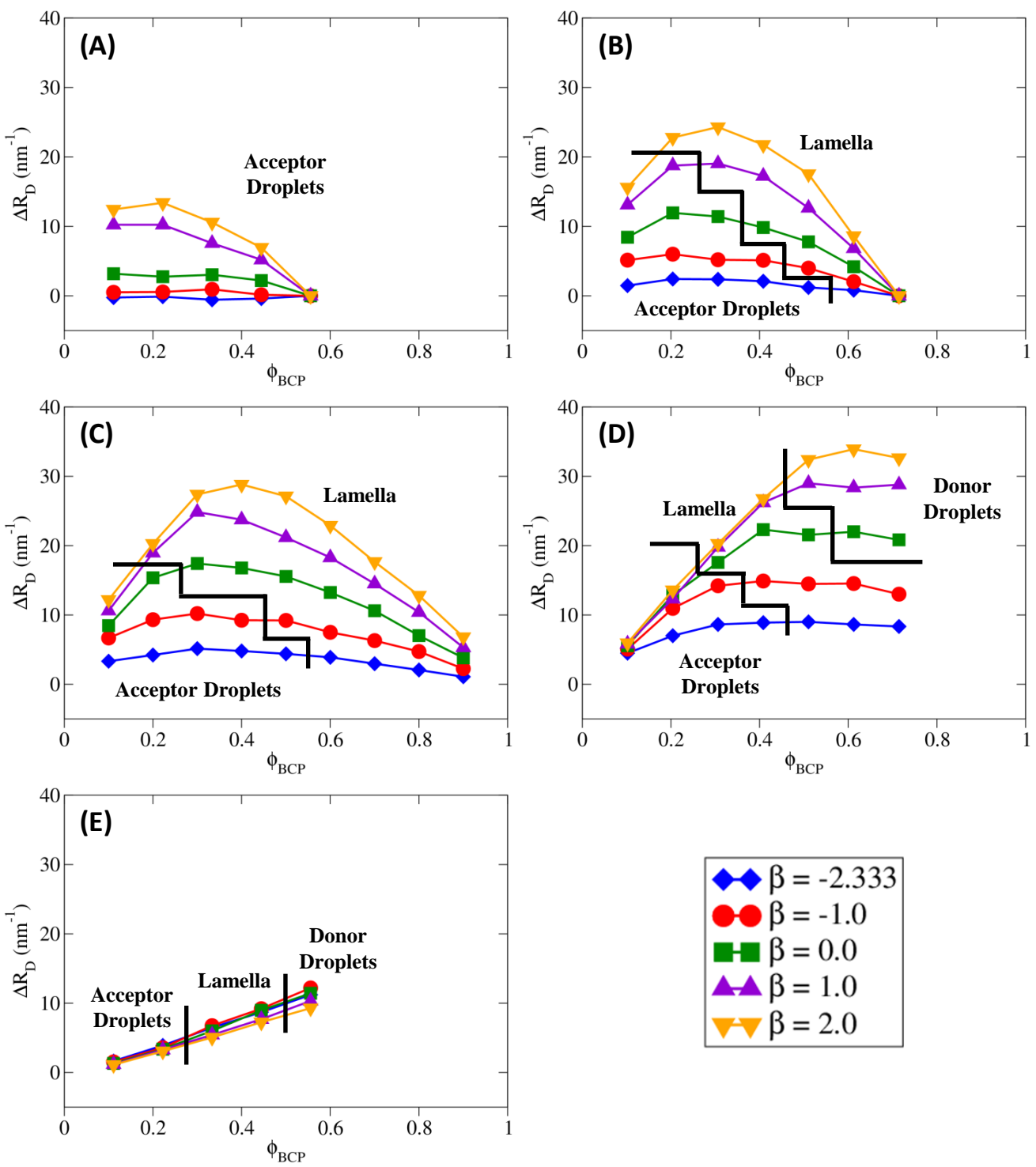

FIG. 9. $\Delta R_{D}$ vs. $\phi_{B C P}$ for varying $\beta$ and $f$ as resulting from $2 \mathrm{D}$ ternary blend morphology simulations. Panels A-F plot results for varying $f$ : (A) $f=0.1,(\mathrm{~B}) f=0.3$, (C) $f=0.5$, (D) $f=0.7$, and (E) $f=0.9$. The solid black lines partition the results into different phases; these lines are approximate and do not represent actual phase boundaries.

the $\mathrm{BCP}$ brought about by the $\mathrm{D} 1$ homopolymer. ${ }^{62}$ For negative $\beta$, the homopolymer D1 is shorter than the BCP A2-b-D2, and the homopolymer swells the D2 brush of the BCP at the interface. Such a condition results in greater degree of intermixing of donors D1 and D2 and a smaller $\Delta R_{D}$.

Increasing $\beta$ causes the homopolymer to increase in length and expel from the D2 brush, 
which reduces the intermixing between D2 and D1, and increases $\Delta R_{D}$. We also observe that $\Delta R_{D}$ changes non-monotonically with increasing $f$. Such a trend can be understood as a result of two competing factors. First, increasing $f$ shortens the D2 brush at the interface, thus expelling the D1 homopolymer and yielding a larger $\Delta R_{D}$. Second, increasing $f$ reduces the blend composition of the D2 donor. Accordingly, a larger $\phi_{B C P}$ is required to achieve the peak in $\Delta R_{D}$. Overall, the maximum $\Delta R_{D}$ results for $\beta=2, f=0.7$, and $\phi_{B C P}=0.6$. However, this blend formulation forms a 2D morphology of donor droplets suspended in acceptor, and so it may not correspond to $X=1$ and $\langle\tau\rangle \approx 1$ in $3 \mathrm{D}$ morphology simulations.

Figure 10 displays $\langle D\rangle$ for varying $\beta, f$, and $\phi_{B C P}$. We observe that $\langle D\rangle$ exhibits irregular trends for both very small and very large $f$. For instance, in the case of $f=0.1$ and for small $\phi_{B C P},\langle D\rangle$ is much larger for $\beta=0,1$, or 2 as compared to $\beta=-1$, or -2.333 . For these blend formulations, the selected $\chi N(\chi N=30)$ is only slightly above $\chi N_{\text {spinodal }}$, and increasing the length of homopolymer D1 chains (increasing $\beta$ ) leads to significantly increased phase separation between D1 and A1, thus yielding a sizable shift in $\langle D\rangle$ from $\beta=0$ to $\beta=1$. In contrast, in the case of $f=0.9$, the domain features approach the size of the simulation box. Accordingly, the $\langle D\rangle$ results exhibit scatter for varying $\beta$ and $\phi_{B C P}$. However, for intermediate values of $f(f=0.3,0.5$, or 0.7$)$, we observe that $\langle D\rangle$ exhibits much more regular trends such that smaller $\langle D\rangle$ results for larger $\beta$, larger $\phi_{B C P}$, and smaller $f$.

In sum, results such as presented in Figures 9 and 10 can be used to discern general targets for the design of ternary blend organic photovoltaics (the results are, however, specific to the system parameters we employed in our simulations). Overall, the results suggest that the optimal blend formulation should incorporate large and positive $\beta$ to target both large $\Delta R_{D}$ and small $\langle D\rangle$. Moreover, intermediate $f(0.3 \leq f \leq 0.7)$ is seen to be optimal to target large $\Delta R_{D}$. A pratical limit to such a strategy is that at large $\beta$, the homopolymer may separate from the blend and form its own macrophase. However, the regimes of occurrance of such a phase transition can be identified based on equilibrium morphology simulations. ${ }^{19}$ For specified combination of $\beta$ and $f$, the volume fraction of block copolymer $\phi_{B C P}$ can then be tuned to target the optimal combination of $\Delta R_{D}$ and $\langle D\rangle$. 

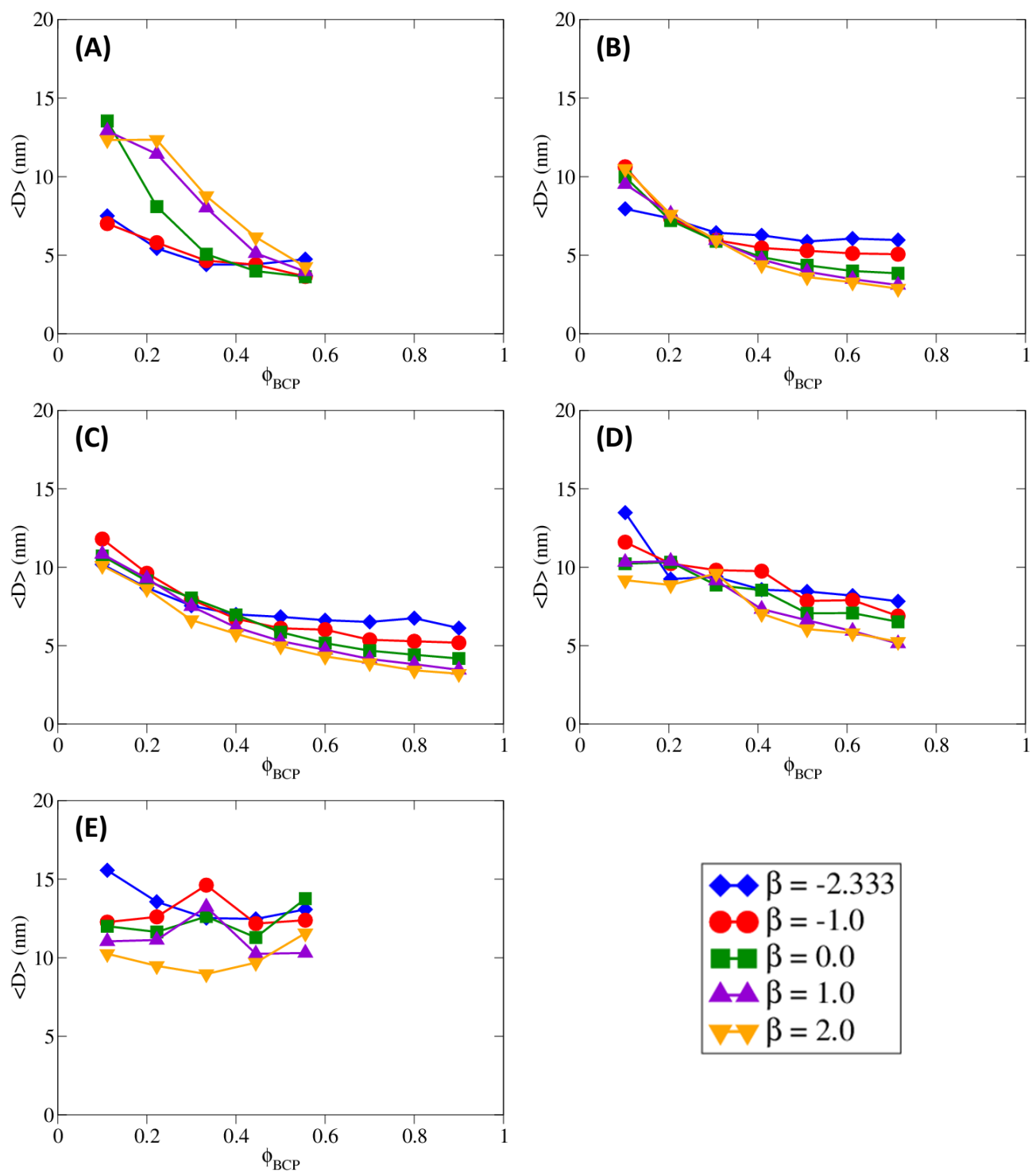

FIG. 10. $\langle D\rangle$ vs. $\phi_{B C P}$ for varying $\beta$ and $f$ as resulting from $2 \mathrm{D}$ ternary blend morphology simulations. Panels A-F plot results for varying $f$ : (A) $f=0.1$, (B) $f=0.3$, (C) $f=0.5$, (D) $f=0.7$, and $(\mathrm{E}) f=0.9$.

\section{Optimizing Device Performance}

While the previous sections dealt with specific device processes (such as exciton dissociation, charge separation, etc.) and identified morphological descriptors that strongly influence such features, the more common device performance features, such as the short-circuit current, $J_{S C}$, and the overall device efficiency, $\eta_{E f f}$, depend on a combination of $\eta_{E D}, \eta_{C S}, F F$, 
and the open circuit voltage, $V_{O C}$. As a consequence, such features are influenced simultaneously by $\langle D\rangle$ and $\Delta R_{D}$. For instance, Figure 11A displays the short-circuit current $J_{S C}$ which is proportional to a product of $\eta_{E D}$ and $\eta_{C S}$. For cases of $\triangle H O M O \neq 0$, we observe that $J_{S C}$ is optimized for $\phi_{B C P}$ that corresponds neither to the peak of $\eta_{E D}$ (i.e. which occurs for the minimum $\langle D\rangle$ ) nor the maximum in $\eta_{C S}$ (which corresponds to the maximum $\Delta R_{D}$ ). Instead, $J_{S C}$ is seen to exhibit a maximum at a $\phi_{B C P}$ that balances the influences of $\eta_{E D}$ and $\eta_{C S}\left(\langle D\rangle\right.$ and $\left.\Delta R_{D}\right)$. Similarly, the device efficiency $\eta_{E f f} \equiv\left(J_{S C} F F V_{O C}\right) / P_{I n}$ (Figure $11 \mathrm{~B}$ ) is optimized for a $\phi_{B C P}$ that balances the influences of $\eta_{E D}, \eta_{C S}, F F$ and $V_{O C}$ (Figure $11 \mathrm{C}$ displays $\left.V_{O C}\right)$.

Notwithstanding the subtleties highlighted in the above paragraph, we suggest that the general considerations discussed in Sections III A - III C, can be equally applied to identify blend formulations that are likely to give rise to improved device performance. To demonstrate this, in Figure $12 \mathrm{~A}$ we combine the $\Delta R_{D}$ and $\langle D\rangle$ results from Figures 9 and 10 into a single figure. We pick three regions and discuss some qualitative considerations that can be deduced based on the findings of the previous sections:

1. Region A delineates three blend formulations that arise for $\beta=0, f=0.5$, and only varying $\phi_{B C P}$. The chosen formulations (i.e. $\phi_{B C P}$ ) represent cases yielding the highest $\Delta R_{D}$ and lowest $D$ for the specified $\beta$ and $f$, and are expected to be the highest performing devices that can be achieved by varying only one blend formulation parameter alone.

2. Region $\mathrm{B}$ delineates three blend formulations that arise by varying $\beta$ and $f$ in addition to $\phi_{B C P}$. Such conditions lead to lamella morphologies with increased $\Delta R_{D}$ and reduced $\langle D\rangle$. Based on the results of the preceding sections, we would expect region $\mathrm{B}$ to give rise to higher $\eta_{E f f}$ as compared to the blend formulations denoted in Region A.

3. Region $\mathrm{C}$ delineates three blend formulations that give rise to the highest $\Delta R_{D}$ overall. The domain sizes for such formulations are comparable to those in Region B. However, these blend formulations also give rise to 2D droplet morphologies and, so, they are also more likely to give rise to sub-unity percolation ratios and increased tortuosity 



FIG. 11. $J_{S C}, \eta_{E f f}$, and $V_{O C}$ results for ternary blends with $\beta=0, f=0.5$, and varying both $\phi_{B C P}$ and $\triangle H O M O$. The optimum $J_{S C}$ and $\eta_{E f f}$ result for a $\phi_{B C P}$ that balances the influence of $\langle D\rangle$ and $\Delta R_{D}$.

in the case of fully 3D morphologies. These blend formulations would be expected to give rise to the highest device performance overall, or they may give rise to inefficient devices due to morphological concerns.

To verify the validity of hypotheses presented above, we conducted fully 3D morphology and device simulations based on the three regions identified in Figure 12A and present the 



FIG. 12. Use of morphological correlators to optimize the device performance of ternary blend organic solar cells. Panel A plots $\Delta R_{D}$ vs $\langle D\rangle$ as resulting from 2D ternary blend morphology simulations. The three dashed ovals in Panel A delineate Regions A, B, and C. Panel B plots $\eta_{E f f}$ as resulting from fully $3 \mathrm{D}$ ternary blend morphology and device simulations. Shown are the average $\eta_{E f f}$ 's resulting from the regions indicated in Panel A.

results of such simulations in Figure 12B. Consistent with our hypothesis, we observe that Regions $\mathrm{B}$ and $\mathrm{C}$ both indeed yield higher $\eta_{E f f}$ than does Region $\mathrm{A}$, and that Region $\mathrm{C}$ yields the highest $\eta_{E f f}$ overall. Indeed, despite yielding more tortuous donor domains as expected, blend formulations in Region $\mathrm{C}$ yield an average $\eta_{E f f}=7.1 \%$. This is in comparison to the blend formulations of Region A, which yield an average $\eta_{E f f}=6.1 \%$. These results suggest that, while the considerations detailed in the previous sections may not necessarily help identify the "optimal" combination of physicochemical parameters, nevertheless, our findings, in conjunction with knowledge of the morphologies, can help screen formulations to discern promising device frameworks.

\section{CONCLUSION}

In this article, we presented results that embody a potential strategy to optimize the device characteristics of bulk heterojunction organic photovoltaics based on ternary blends containing BCP compatibilizers. Towards this goal, we identified a host of morphological descriptors that correlate to the device characteristics of ternary blend organic solar cells based on block copolymer additives. Based on these findings, we identified that the domain percolation ratio, tortuosity, and size, as well as the concentration gradient at the interface 
between donors and acceptors, correlate strongly with the device characteristics, and can therefore be considered as morphological correlators for device performance. Such findings were then used in conjunction with a morphological simulation tool to identify optimal (in morphological descriptors) blend formulations when the blend composition, the degree of polymerization of the donor homopolymer, and the acceptor composition of the donor- $b$ acceptor block copolymer are simultaneously varied. Through such a process, we identified that long homopolymer donors and slightly assymetric BCPs with higher donor compositions give rise to the optimal morphological correlators and, indeed, the highest device efficiencies overall.

While the quantitative aspects of the results presented in this paper are tied to the specific parameters and the model adopted, many of the general considerations so-deduced transcend such details. An important finding from our work was the role of a new morphological measure, $\Delta R_{D}$, that quantifies the strength of energy cascades and its influence upon charge separation and fill factor. Secondly, we found that, in the presence of energy cascades, $\Delta R_{D}$ has a much stronger effect on photophysical processes compared to the influence of domain sizes, $\langle D\rangle$. Finally, we demonstrated the manner in which morphological descriptors can be integrated with morphology prediction tools to provide a complete framework for in-silco computational design and optimization of such ternary blends. Such a framework can potentially be adapted to the study of other organic ternary blends which have recently attracted significant attention. ${ }^{32-36}$

\section{ACKNOWLEDGMENTS}

This work was supported in part by grants from the Robert A. Welch Foundation (Grant F1599), the National Science Foundation (CBET-1264583, CMMI-1149365), and the US

Army Research Office (W911NF-13-1-0396). The authors acknowledge the Texas Advanced Computing Center (TACC) at The University of Texas at Austin for providing computing 
resources that have contributed to the research results reported within this paper.

*venkat@che.utexas.edu

1 Christoph J. Brabec, Martin Heeney, Iain McCulloch, and Jenny Nelson. Influence of blend microstructure on bulk heterojunction organic photovoltaic performance. Chemical Society Reviews, 40(3):1185-1199, 2011.

2 Christoph J. Brabec, Srinivas Gowrisanker, Jonathan J. M. Halls, Darin Laird, Shijun Jia, and Shawn P. Williams. Polymer-Fullerene Bulk-Heterojunction Solar Cells. Adv. Mater., 22(34):3839-3856, 2010.

3 Barry C. Thompson and Jean M. J. Frechet. Polymer-Fullerene Composite Solar Cells. Angewandte Chemie International Edition, 47(1):58-77, 2008.

4 L. Y. Lu, T. Y. Zheng, Q. H. Wu, A. M. Schneider, D. L. Zhao, and L. P. Yu. Recent advances in bulk heterojunction polymer solar cells. Chemical Reviews, 115(23):12666-12731, December 2015.

5 Mikkel Jorgensen, Kion Norrman, Suren A. Gevorgyan, Thomas Tromholt, Birgitta Andreasen, and Frederik C. Krebs. Stability of polymer solar cells. Advanced Materials, 24(5):580-612, February 2012.

6 Y. Kim, S. A. Choulis, J. Nelson, D. D. C. Bradley, S. Cook, and J. R. Durrant. Device annealing effect in organic solar cells with blends of regioregular poly(3-hexylthiophene) and soluble fullerene. Applied Physics Letters, 86(6):063502, February 2005.

7 Tao Wang, Andrew J. Pearson, David G. Lidzey, and Richard A. L. Jones. Evolution of structure, optoelectronic properties, and device performance of polythiophene: Fullerene solar cells during thermal annealing. Advanced Functional Materials, 21(8):1383-1390, April 2011.

8 Matthew O. Reese, Alexandre M. Nardes, Benjamin L. Rupert, Ross E. Larsen, Dana C. Olson, Matthew T. Lloyd, Sean E. Shaheen, David S. Ginley, Garry Rumbles, and Nikos Kopidakis. Photoinduced degradation of polymer and polymer-fullerene active layers: Experiment and theory. Advanced Functional Materials, 20(20):3476-3483, October 2010. 
9 Yong Hyun Kim, Christoph Sachse, Michael L. Machala, Christian May, Lars Mueller-Meskamp, and Karl Leo. Highly conductive pedot:pss electrode with optimized solvent and thermal posttreatment for ito-free organic solar cells. Advanced Functional Materials, 21(6):1076-1081, March 2011.

10 Tao Wang, Alan D. F. Dunbar, Paul A. Staniec, Andrew J. Pearson, Paul E. Hopkinson, J. Emyr MacDonald, Samuele Lilliu, Claire Pizzey, Nicholas J. Terrill, Athene M. Donald, Anthony J. Ryan, Richard A. L. Jones, and David G. Lidzey. The development of nanoscale morphology in polymer: fullerene photovoltaic blends during solvent casting. Soft Matter, 6(17):4128-4134, 2010.

11 Hongping Yan, Sufal Swaraj, Cheng Wang, Inchan Hwang, Neil C. Greenham, Chris Groves, Harald Ade, and Christopher R. McNeill. Influence of annealing and interfacial roughness on the performance of bilayer donor/acceptor polymer photovoltaic devices. Advanced Functional Materials, 20(24):4329-4337, December 2010.

12 Hin-Lap Yip and Alex K. . Y. Jen. Recent advances in solution-processed interfacial materials for efficient and stable polymer solar cells. Energy \& Environmental Science, 5(3):5994-6011, March 2012.

13 Sabine Bertho, Ilse Haeldermans, Ann Swinnen, Wouter Moons, Tom Martens, Laurence Lutsen, Dirk Vanderzande, Jean Manca, Alessia Senes, and Annalisa Bonfiglio. Influence of thermal ageing on the stability of polymer bulk heterojunction solar cells. Solar Energy Materials and Solar Cells, 91(5):EU Project MOLYCELL, March 2007.

14 M. C. Chen, D. J. Liaw, Y. C. Huang, H. Y. Wu, and Y. Tai. Improving the efficiency of organic solar cell with a novel ambipolar polymer to form ternary cascade structure. Solar Energy Materials and Solar Cells, 95(9):2621-2627, September 2011.

15 Sebastien Lizin, Steven Van Passel, Ellen De Schepper, Wouter Maes, Laurence Lutsen, Jean Manca, and Dirk Vanderzande. Life cycle analyses of organic photovoltaics: a review. Energy E Environmental Science, 6(11):3136-3149, November 2013.

16 K. Sivula, Z. T. Ball, N. Watanabe, and J. M. J. Frechet. Amphiphilic Diblock Copolymer Compatibilizers and Their Effect on the Morphology and Performance of Polythiophene:Fullerene Solar Cells. Advanced Materials, 18(2):206-210, 2006. 
17 Dylan Kipp, Olga Wodo, Baskar Ganapathysubramanian, and Venkat Ganesan. Achieving Bicontinuous Microemulsion Like Morphologies in Organic Photovoltaics. ACS Macro Letters, $4(2): 266-270,2015$.

18 Dylan Kipp, Jorge Mok, Joseph Strzalka, Seth B. Darling, Venkat Ganesan, and Rafael Verduzco. Rational Design of Thermally Stable, Bicontinuous Donor/Acceptor Morphologies with Conjugated Block Copolymer Additives. ACS Macro Letters, 4(9):867-871, 2015.

19 Dylan Kipp, Rafael Verduzco, and Venkat Ganesan. Design of bicontinuous donor/acceptor morphologies for use as organic solar cell active layers. Journal of Polymer Science Part B: Polymer Physics, pages n/a-n/a, 2016.

20 Dylan Kipp and Venkat Ganesan. Exploiting the combined influence of morphology and energy cascades in ternary blend organic solar cells based on block copolymer additives. Macromolecules, 49(14):5137-5144, 2016.

21 Sean E. Shaheen, Christoph J. Brabec, N. Serdar Sariciftci, Franz Padinger, Thomas Fromherz, and Jan C. Hummelen. 2.5\% efficient organic plastic solar cells. Appl. Phys. Lett., 78(6):841-843, 2001.

22 H. Hoppe, M. Niggemann, C. Winder, J. Kraut, R. Hiesgen, A. Hinsch, D. Meissner, and N.?S. Sariciftci. Nanoscale Morphology of Conjugated Polymer/Fullerene-Based Bulk- Heterojunction Solar Cells. Adv. Funct. Mater., 14(10):1005-1011, 2004.

23 Harald Hoppe and Niyazi Serdar Sariciftci. Morphology of Polymer/Fullerene Bulk Heterojunction Solar Cells. J. Mater. Chem., 16:45-61, 2006.

24 Dian Chen, Atsuhiro Nakahara, Dongguang Wei, Dennis Nordlund, and Thomas P. Russell. P3HT/PCBM Bulk Heterojunction Organic Photovoltaics: Correlating Efficiency and Morphology. Nano Lett., 11(2):561-567, 2011.

25 Wei Chen, Tao Xu, Feng He, Wei Wang, Cheng Wang, Joseph Strzalka, Yun Liu, Jianguo Wen, Dean J. Miller, Jihua Chen, Kunlun Hong, Luping Yu, and Seth B. Darling. Hierarchical Nanomorphologies Promote Exciton Dissociation in Polymer/Fullerene Bulk Heterojunction Solar Cells. Nano Lett., 11(9):3707-3713, 2011.

26 Feng Liu, Yu Gu, Jae Woong Jung, Won Ho Jo, and Thomas P. Russell. On the Morphology of Polymer-Based Photovoltaics. J. Polym. Sci. Part B. Polym. Phys., 50(15):1018-1044, 2012. 
27 Bingyuan Huang, Jojo A. Amonoo, Anton Li, X. Chelsea Chen, and Peter F. Green. Role of Domain Size and Phase Purity on Charge Carrier Density, Mobility, and Recombination in Poly(3-hexylthiophene):Phenyl-C61-butyric Acid Methyl Ester Devices. The Journal of Physical Chemistry C, 118(8):3968-3975, 2014.

Olga Wodo, Srikanta Tirthapura, Sumit Chaudhary, and Baskar Ganapathysubramanian. Computational characterization of bulk heterojunction nanomorphology. J. Appl. Phys., 112(6):-, 2012 .

29 Olga Wodo, Srikanta Tirthapura, Sumit Chaudhary, and Baskar Ganapathysubramanian. A graph-based formulation for computational characterization of bulk heterojunction morphology. Organic Electronics, 13(6):1105 - 1113, 2012.

30 Olga Wodo, John D. Roehling, Adam J. Moule, and Baskar Ganapathysubramanian. Quantifying organic solar cell morphology: a computational study of three-dimensional maps. Energy Environ. Sci., 6:3060-3070, 2013.

31 Olga Wodo, Jaroslaw Zola, Balaji Sesha Sarath Pokuri, Pengfei Du, and Baskar Ganapathysubramanian. Automated, high throughput exploration of process-structure-property relationships using the mapreduce paradigm. Materials Discovery, 1:21-28, 2015.

32 Tayebeh Ameri, Parisa Khoram, Jie Min, and Christoph J. Brabec. Organic Ternary Solar Cells: A Review. Advanced Materials, 25(31):4245-4266, 2013.

33 Qiaoshi An, Fujun Zhang, Jian Zhang, Weihua Tang, Zhenbo Deng, and Bin Hu. Versatile ternary organic solar cells: a critical review. Energy $\&$ Environmental Science, 9(2):281-322, 2016.

34 Luyao Lu, Mary Allison Kelly, Wei You, and Luping Yu. Status and prospects for ternary organic photovoltaics. Nature Photonics, 9(8):491-500, August 2015.

35 Tao Liu, Lijun Huo, Xiaobo Sun, Bingbing Fan, Yunhao Cai, Taehyo Kim, Jin Young Kim, Hyosung Choi, and Yanming Sun. Ternary organic solar cells based on two highly efficient polymer donors with enhanced power conversion efficiency. Advanced Energy Materials, 6(6):1502109, March 2016.

36 Brett M. Savoie, Scott Dunaisky, Tobin J. Marks, and Mark A. Ratner. The scope and limitations of ternary blend organic photovoltaics. Advanced Energy Materials, 5(3):1400891, February 2015. 
L. Y. Lu, T. Xu, W. Chen, E. S. Landry, and L. P. Yui. Ternary blend polymer solar cells with enhanced power conversion efficiency. Nature Photonics, 8(9):716-722, September 2014.

38 L. Y. Lu, W. Chen, T. Xu, and L. P. Yu. High-performance ternary blend polymer solar cells involving both energy transfer and hole relay processes. Nature Communications, 6:7327, June 2015.

39 Dylan Kipp and Venkat Ganesan. Influence of Block Copolymer Compatibilizers on the Morphologies of Semiflexible Polymer/Solvent Blends. J. Phys. Chem. B, 118(16):4425-4441, 2014.

40 Dylan Kipp and Venkat Ganesan. A Kinetic Monte Carlo Model with Improved Charge Injection Model for the Photocurrent Characteristics of Organic Solar Cells. J. Appl. Phys., 113(23):-, 2013.

41 Harald Hoppe and Niyazi Serdar Sariciftci. Organic solar cells: An overview. J. Mater. Res., 19:1924-1945, 2004.

42 Armin Schneider, Jean-Jacques Zanna, Masayuki Yamada, Heino Finkelmann, and Ralf Thomann. Competition between Liquid Crystalline Phase Symmetry and Microphase Morphology in a Chiral Smectic Liquid Crystalline-Isotropic Block Copolymer. Macromolecules, 33(3):649-651, 2000.

43 M. Lee, J.-W. Kim, I.-W. Hwang, Y.-R. Kim, N.-K. Oh, and W.-C. Zin. Nanoscale Organization of Conjugated Rods in Rod-Coil Molecules. Adv. Mater., 13(18):1363-1368, 2001.

44 L. H. Radzilowski, J. L. Wu, and S. I. Stupp. Monodisperse "Rodcoil" Copolymers. Macromolecules, 26(4):879-882, 1993.

45 L. H. Radzilowski and S. I. Stupp. Nanophase Separation in Monodisperse Rodcoil Diblock Polymers. Macromolecules, 27(26):7747-7753, 1994.

46 L. H. Radzilowski, B. O. Carragher, and S. I. Stupp. Three-Dimensional Self-Assembly of Rodcoil Copolymer Nanostructures. Macromolecules, 30(7):2110-2119, 1997.

47 N. Arun Kumar and Venkat Ganesan. Communication: Self-Assembly of Semiflexible-Flexible Block Copolymers. J. Chem. Phys., 136(10):101101, 2012.

48 Yongye Liang, Zheng Xu, Jiangbin Xia, Szu-Ting Tsai, Yue Wu, Gang Li, Claire Ray, and Luping Yu. For the Bright Future - Bulk Heterojunction Polymer Solar Cells with Power Conversion Efficiency of 7.4 Adv. Mater., 22(20):E135-E138, 2010. 
49 Petr P. Khlyabich, Beate Burkhart, and Barry C. Thompson. Efficient Ternary Blend Bulk Heterojunction Solar Cells with Tunable Open-Circuit Voltage. Journal of the American Chemical Society, 133(37):14534-14537, 2011. PMID: 21854034.

50 Petr P. Khlyabich, Andrey E. Rudenko, Barry C. Thompson, and Yueh-Lin Loo. Structural Origins for Tunable Open-Circuit Voltage in Ternary-Blend Organic Solar Cells. Advanced Functional Materials, 25(34):5557-5563, 2015.

51 Cheng-Kuang Lee, Olga Wodo, Baskar Ganapathysubramanian, and Chun-Wei Pao. Electrode materials, thermal annealing sequences, and lateral/vertical phase separation of polymer solar cells from multiscale molecular simulations. Acs Applied Materials \& Interfaces, 6(23):2061220624, December 2014.

52 Chris Groves. Suppression of geminate charge recombination in organic photovoltaic devices with a cascaded energy heterojunction. Energy Environ. Sci., 6:1546-1551, 2013.

53 G. Fredrickson. The Equilibrium Theory of Inhomogeneous Polymers. Oxford University Press, 2006.

54 Kostas Ch. Daoulas and Marcus Müller. Single Chain in Mean Field Simulations: QuasiInstantaneous Field Approximation and Quantitative Comparison with Monte Carlo Simulations. J. Chem. Phys., 125(18):184904, 2006.

55 Gunja Pandav and Venkat Ganesan. Efficacy of Different Block Copolymers in Facilitating Microemulsion Phases in Polymer Blend Systems. Macromolecules, 46(20):8334-8344, 2013.

56 Gunja Pandav and Venkat Ganesan. Fluctuation Effects on the Order-Disorder Transition in Polydisperse Copolymer Melts. J. Chem. Phys., 139(21):214905, 2013.

57 Matthew L. Jones, Reesha Dyer, Nigel Clarke, and Chris Groves. Are hot charge transfer states the primary cause of efficient free-charge generation in polymer:fullerene organic photovoltaic devices? A kinetic Monte Carlo study. Phys. Chem. Chem. Phys., 16:20310-20320, 2014.

58 Hari K Kodali and Baskar Ganapathysubramanian. Sensitivity analysis of current generation in organic solar cellscomparing bilayer, sawtooth, and bulk heterojunction morphologies. Solar Energy Materials and Solar Cells, 111:66-73, 2013.

59 Hari K Kodali and Baskar Ganapathysubramanian. Computer simulation of heterogeneous polymer photovoltaic devices. Modelling and Simulation in Materials Science and Engineering, 
20(3):035015, 2012.

60 Hari K Kodali and Baskar Ganapathysubramanian. A computational framework to investigate charge transport in heterogeneous organic photovoltaic devices. Computer Methods in Applied Mechanics and Engineering, 247:113-129, 2012.

61 Thomas Kirchartz and Jenny Nelson. Device modelling of organic bulk heterojunction solar cells. Multiscale Modelling of Organic and Hybrid Photovoltaics, 352:279-324, 2014.

62 L. Leibler. Emulsifying Effects of Block Copolymers in Incompatible Polymer Blends. Makromol. Chem. Macromol. Symp., 16:1-17, 1988. 